\title{
Bortezomib Treatment for Refractory PLA2R-Positive Membranous Nephropathy
}

\author{
Abdallah S. Geara ${ }^{a}$ Vijay Bhoj ${ }^{b}$ Jonathan J. Hogan ${ }^{a}$ \\ aDivision of Nephrology, Perelman School of Medicine, University of Pennsylvania, Philadelphia, PA, USA; \\ ${ }^{b}$ Department of Pathology and Laboratory Medicine, Perelman School of Medicine, University of Pennsylvania, \\ Philadelphia, PA, USA
}

\section{Keywords}

Membranous nephropathy · Glomerulonephritis ·

Nephrotic syndrome

\begin{abstract}
Introduction: B-cell depletion has been shown to be an effective strategy for the majority of patients with membranous nephropathy (MN), and in PLA2R-positive MN, immunologic remission (improvement or elimination of measurable serum anti-PLA2R antibodies) precedes renal remission. Yet, cases exist of patients who do not achieve immunologic remission despite achieving peripheral B-cell depletion. This has led to the hypothesis that some patients have plasma cells that are responsible for producing anti-PLA2R antibodies. Case Presentation: A 66-year-old man with a past medical history of hypertension, hyperlipidemia, and cerebrovascular disease presented with nephrotic syndrome and was diagnosed with PLA2R-positive MN on kidney biopsy. He was refractory to multiple therapies including tacrolimus, and was resistant to rituximab despite having achieved Bcell depletion. He also did not enter into remission with plasmapharesis and cyclophosphamide. He then achieved immediate immunologic remission after treatment with the proteasome inhibitor bortezomib, which is used as first-line
\end{abstract}

karger@karger.com www.karger.com/gdz

Karger $\stackrel{\text { ' }}{=}$

GOPEN ACCESS
(C) 2021 The Author(s)

Published by S. Karger AG, Basel

This is an Open Access article licensed under the Creative Commons Attribution-NonCommercial-4.0 International License (CC BY-NC) (http://www.karger.com/Services/OpenAccessLicense), applicable to the online version of the article only. Usage and distribution for commercial purposes requires written permission. therapy for multiple myeloma. Discussion/Conclusion: This case suggests that considering the source of PLA2R antibody production could lead to individualized and targeted therapies for MN.

(C) 2021 The Author(s)

Published by S. Karger AG, Basel

\section{Introduction}

B-cell depletion is an effective treatment strategy for most cases of membranous nephropathy (MN), and in PLA2R positive $M N$, improvement in serum anti-PLA2R antibody levels precedes remission in proteinuria $[1,2]$. Cases of MN that are refractory to B-cell depletion, and higher remission rates observed with cyclophosphamide versus rituximab $[3,4]$, have led to the hypothesis some patients have long-lived plasma cells that are responsible for anti-PLA2R antibody production, and that these patients may be candidates for anti-plasma cell agents such as bortezomib. We report a patient with PLA2R-positive $\mathrm{MN}$ who was refractory to B-cell depletion, but who achieved immediate immunologic remission after treatment with bortezomib, a proteasome inhibitor that is used as first-line anti-plasma therapy for multiple myeloma. 


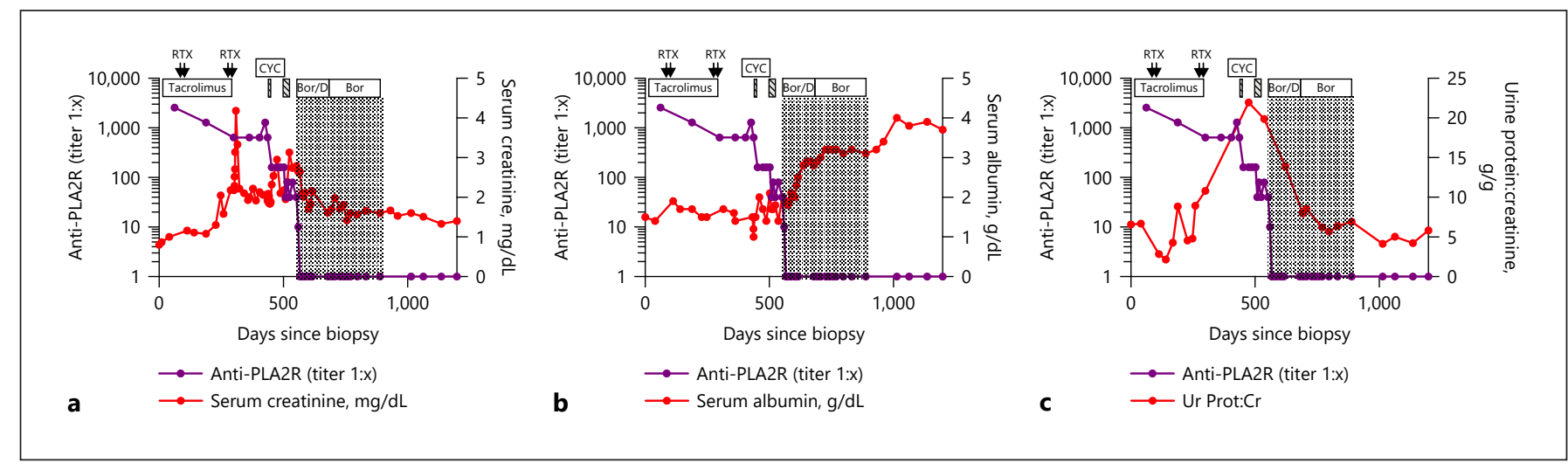

Fig. 1. Treatment courses and laboratory trends for serum anti-PLA2R antibody and serum creatinine (a), serum albumin (b), and proteinuria (c). Striped boxes indicate courses of plasmapheresis. RTX, rituximab; CYC, cyclophosphamide; Bor/D-bortezomib/dexamethasone.

\section{Case Presentation}

A 66-year-old man with a past medical history of hypertension, hyperlipidemia, and cerebrovascular disease presented with severe nephrotic syndrome and was diagnosed with PLA2R-positive MN on kidney biopsy. Serum anti-PLA2R antibody was markedly elevated at 1:2,560 by indirect immunofluorescence testing (IFF, negative $<1: 10$ ). Figure 1 illustrates his treatment and follow-up. After initial treatment with tacrolimus and prednisone, he received 2 doses of intravenous rituximab 1,000 mg biweekly which was repeated again at 6 months. He was found to have severe hypogammaglobulinemia but did not develop any infections. Despite achieving complete peripheral B-cell depletion (CD19 0\%), he remained severely nephrotic and serum anti-PLA2R antibody remained elevated (1:640 by IIF, $692 \mathrm{RU} / \mathrm{mL}$ by ELISA [normal $<19 \mathrm{RU} / \mathrm{mL}]$ ). He was then treated with biweekly intravenous cyclophosphamide (chosen due to severe nausea and anorexia attributed to gastrointestinal tract edema) with dose escalation until he developed neutropenia. He did not experience any other adverse events with cyclophosphamide. Despite continued peripheral Bcell depletion (CD19 0\%), serum anti-PLA2R antibody remained markedly positive (1:640-1:1,280). He also underwent 2 courses of plasmapharesis in an attempt to remove the pathogenic antiPLA2R antibody. One month after his last cyclophosphamide dose, and while on plasmapheresis, his serum anti-PLA2R antibody remained positive at 1:40. One month after stopping plasmapheresis, his serum anti-PLA2R antibody remained positive at 1:40-1:80 with continued severe nephrotic syndrome. He also received subcutaneous immunoglobulin for his severe hypogammaglobulinemia.

One month after stopping plasmapheresis, and 2 months after his last cyclophosphamide dose, he started anti-plasma cell therapy with weekly bortezomib $\left(1.3 \mathrm{mg} / \mathrm{m}^{2}\right)$ and dexamethasone $(20$ mg weekly). One week after starting therapy, his serum antiPLA2R antibody had decreased by 2 dilutions, from 1:40 to 1:10, and 2 weeks after starting therapy, his serum anti-PLAR was undetectable $(<1: 10)$. His serum creatinine also stabilized. He was continued on weekly bortezomib and tapered off dexamethasone over 4 months. His bortezomib dosing was changed to every other

Bortezomib Treatment for Refractory

Membranous Nephropathy week dosing after 12 weekly doses, and then to monthly dosing after 14 more weeks of therapy, then at which point it was stopped (total duration of bortezomib was 11 months). His serum antiPLA2R antibody remained negative by both indirect immunofluorescence test $(<1: 10)$ and ELISA $(<1.8 \mathrm{RU} / \mathrm{mL})$ during this time. At last follow-up, 21 months after starting bortezomib and 9 months after discontinuation of all immunosuppression, his kidney function had improved to ( $\mathrm{SCr} 1.4 \mathrm{mg} / \mathrm{dL}$ ), his serum albumin normalized (3.7-4.0 g/dL), and his proteinuria has continued to improve (best value $4.1 \mathrm{~g} / \mathrm{g}$ ) (Fig. 1). His hypogammaglobulinemia improved with subcutaneous immunoglobulin therapy which was being tapered, and his CD19 cells had recovered (8\% [normal 6-29\%], absolute count $176 / \mu \mathrm{L}$ [normal $110-660 / \mu \mathrm{L}])$. His serum anti-PLA2R antibody remains undetectable by IIF.

\section{Discussion}

In PLA2R-positive $\mathrm{MN}$, a renal response depends on achieving immunologic response (i.e., improvement or disappearance of measurable serum anti-PLA2R antibody), which can precede improvements in proteinuria and serum albumin by weeks to months. For decades, the first-line treatment for $\mathrm{MN}$ has been cyclophosphamidebased regimens, which are known to be effective for achieving both immunologic and renal remission. However, additional treatment options for $\mathrm{MN}$ are needed due to cyclophosphamide-related toxicity [3], particularly in patients who require multiple treatment courses, as well as for patients who are non-responders to cyclophosphamide.

The Membranous Nephropathy Trial of Rituximab (MENTOR trial) confirmed substantial uncontrolled data showing that $\mathrm{B}$-cell depletion with rituximab is a 
more effective strategy for the treatment of $\mathrm{MN}$ than cyclosporine $[1,2]$. These results are expected to be incorporated into the next iteration of the Kidney Disease: Improving Global Outcomes Clinical Practice Guidelines for Glomerulonephritis for the management of MN. The Sequential Treatment with Tacrolimus-Rituximab versus Steroids plus Cyclophosphamide in Patients with Primary Membranous Nephropathy (STARMEN) study also shed further light on the efficacy and safety of cyclophosphamide versus rituximab-based regimens [5].

However, there is an unmet need for additional treatment strategies in patients who do not tolerate these therapies, do not achieve immunologic remission with these therapies, and/or develop anti-rituximab antibodies. Case reports have shown that the "next-generation" antiCD20 antibodies obinutuzumab [6] and ofatumumab [79] may be promising agents in such cases. Successful treatment of rituximab-resistant patients with obinutuzumab and ofatumumab could imply that additional or more complete B-cell depletion is required in such cases to achieve immunologic remission, but also that the producing anti-PLA2R antibody-producing cell is a CD20positive B cell.

An alternative hypothesis in patients who do not achieve immunologic remission despite achieving peripheral B-cell depletion is that these patients have longlived plasma cells that are responsible for anti-PLA2R antibody production. This was the rationale for prescribing the proteasome inhibitor bortezomib, which is a first-line therapy for multiple myeloma, to our patient who presented with severe PLA2R positive MN which was resistant to multiple therapies despite documented peripheral $\mathrm{B}$-cell depletion. There is also an active clinical trial evaluating anti-CD38 antibody treatments to target plasma cells in PLA2R-associated MN (NCT0415440).

This case suggests that anti-plasma cell therapy may be useful in select cases of MN that are immunologically active despite successful achieving B-cell depletion with rituximab and/or cyclophosphamide. Two previous case reports describe the use of bortezomib in $\mathrm{MN}$ : one in a patient with $\mathrm{MN}$ after kidney transplant whose proteinuria improved with bortezomib $\left(1.3 \mathrm{mg} / \mathrm{m}^{2}, 4\right.$ doses over 2 weeks) after having failed rituximab therapy [10], and a second of a patient with severe nephrotic syndrome due to $\mathrm{MN}$ who experienced a complete remission in proteinuria with bortezomib therapy $\left(1.6 \mathrm{mg} / \mathrm{m}^{2}\right.$ on days $1,4,8$, and 11) after having failed treatment with high dose steroids and ramipril [11]. Both of these cases showed impressive clinical responses to bortezomib, but lacked PLA2R antigen testing on the kidney biopsy and serum
anti-PLA2R antibody levels for assessment of immunologic response.

The interpretation of our case report has some important limitations. Most measurements of serum antiPLA2R levels were performed by indirect immunofluorescence test, which is a semi-quantitative test, rather than the quantitative ELISA assay. Moreover, the timing of our patient entering remission with bortezomib-dexamethasone may be confounded by prior cyclophosphamide and plasmapheresis treatments.

The dose and duration of bortezomib therapy required to induce an immunologic remission also require further study. As noted previously, the prior 2 case reports prescribed 4 doses of $1.3-1.6 \mathrm{mg} / \mathrm{m}^{2}$ within a 4 -week period. We prescribed a long course of bortezomib therapy given the significant comorbidities that he had experienced while nephrotic, and his gradual improvement in rehabilitation, quality of life, and volume status after having achieved immunologic remission. He was tolerating bortezomib well and he agreed that a relapse would have been severely detrimental. Bortezomib must be prescribed with caution and close monitoring given that it is known to cause severe neuropathy in some patients. Monitoring of plasma cells in the peripheral blood, as had been described in one of prior case report [10], in addition to B cells and anti-PLA2R antibody titers may assist with individualizing bortezomib treatment to avoid over-exposure to the drug.

Notwithstanding these limitations, the rapid and sustained remission that our patient experienced with bortezomib after having failed multiple prior therapies, and precedent for the use of anti-plasma cell therapies in other antibody-mediated diseases including $\mathrm{MN}$, suggest that further study of B- and plasma cell repertoires may help developed individualized and targeted treatment strategies for $\mathrm{MN}$.

\section{Statement of Ethics}

This research was conducted ethically and in accordance with the World Medical Association Declaration of Helsinki. We have received written (electronically) consent from the patient presented in this report to publish the medical details of his case. No protected health information or personal identifying information is included in the manuscript.

\section{Conflict of Interest Statement}

J.J.H. reports having received consulting fees from G.S.K., Alexion, Retrophin, Zyversa, Dimerix, and Goldfinch Bio. He also reports salary support through grant funding as site principle in-
Geara/Bhoj/Hogan 
vestigator for studies from the National Institutes of Health, Gilead, G.S.K., Regeneron, Boehringer Ingelheim, Retrophin, Achillion, Calliditas, Omeros, and BMS. He receives royalties from UpToDate.com for topics written on calcium-phosphorous balance and monoclonal gammopathies of renal significance. He also is a co-inventor and has received royalties related to a pending patent involving the use of synthetic immunoreceptor engineered $\mathrm{T}$ cells for the treatment of membranous nephropathy. V.B. is a co-inventor and has received royalties related to patents involving the use of synthetic immunoreceptor engineered T cells for the treatment of cancer and antibody-mediated diseases. A.S.G. has no conflicts of interests to declare. None of the above conflicts of interest are relevant to this work. This manuscript is not currently under consideration for publication at any other journal and has not been previously published.

\section{Funding Sources}

The authors did not receive any funding.

\section{Author Contributions}

A.S.G. contributed to manuscript writing and review. V.G.B. contributed to manuscript writing, figure preparation, and review. J.J.H. contributed to manuscript writing and review.

\section{References}

1 Ruggenenti P, Cravedi P, Chianca A, Perna A, Ruggiero B, Gaspari F, et al. Rituximab in idiopathic membranous nephropathy. J Am Soc Nephrol. 2012;23(8):1416-25.

2 Fervenza FC, Appel GB, Barbour SJ, Rovin BH, Lafayette RA, Aslam N, et al. Rituximab or cyclosporine in the treatment of membranous nephropathy. N Engl J Med. 2019; 381(1):36-46.

3 van den Brand JAJG, Ruggenenti P, Chianca A, Hofstra JM, Perna A, Ruggiero B, et al. Safety of rituximab compared with steroids and cyclophosphamide for idiopathic membranous nephropathy. J Am Soc Nephrol. 2017;28(9):2729-37.

4 van de Logt AE, Dahan K, Rousseau A, van der Molen R, Debiec H, Ronco P, et al. Immunological remission in PLA2R-antibodyassociated membranous nephropathy: cyclophosphamide versus rituximab. Kidney Int. 2018;93(4):1016-7.
5 Fernandez-Juarez G, Rojas-Rivera J, Logt AV, Justino J, Sevillano A, Caravaca-Fonta'n F, et al. The STARMEN trial indicates that alternating treatment with corticosteroids and cyclophosphamide is superior to sequential treatment with tacrolimus and rituximab in primary membranous nephropathy. Kidney Int. Forthcoming 2020.

6 Klomjit N, Fervenza FC, Zand L. Successful treatment of patients with refractory PLA2Rassociated membranous nephropathy with obinutuzumab: a report of 3 cases. Am J Kidney Dis. 2020 Dec 1;76(6):P883-8.

7 Podesta MA, Gennarini A, Portalupi V, Rota S, Alessio MG, Remuzzi G, et al. Accelerating the depletion of circulating anti-phospholipase $\mathrm{A} 2$ receptor antibodies in patients with severe membranous nephropathy: preliminary findings with double filtration plasmapheresis and ofatumumab. Nephron. 2020; 144:30-5.
8 Podesta MA, Ruggiero B, Remuzzi G, Ruggenenti P. Ofatumumab for multirelapsing membranous nephropathy complicated by rituximab-induced serum-sickness. BMJ Case Rep. 2020 Jan 23;13.

9 Boyer-Suavet S, Andreani M, Lateb M, Savenkoff B, Brglez V, Benzaken S, et al. Neutralizing anti-rituximab antibodies and relapse in membranous nephropathy treated with rituximab. Front Immunol. 2019;10:3069.

10 Barbari A, Chehadi R, Kfoury Assouf H, Kamel G, Jaafar M, Abdallah A, et al. Bortezomib as a novel approach to early recurrent membranous glomerulonephritis after kidney transplant refractory to combined conventional rituximab therapy. Exp Clin Transpl. 2017;15(3):350-4.

11 Hartono C, Chung M, Kuo SF, Seshan SV, Muthukumar T. Bortezomib therapy for nephrotic syndrome due to idiopathic membranous nephropathy. J Nephrol. 2014;27(1): 103-6. 